\title{
ERROR BOUNDS FOR THE NUMERICAL EVALUATION OF LEGENDRE POLYNOMIALS BY A THREE-TERM RECURRENCE*
}

\author{
TOMASZ HRYCAK $^{\dagger}$ AND SEBASTIAN SCHMUTZHARD ${ }^{\dagger}$
}

Abstract. We study the numerical evaluation of the Legendre polynomials $P_{n}$ on the interval $[-1,1]$ via a three-term recurrence. We prove that in a neighborhood of an endpoint, the computed approximation exactly agrees with the line tangent to $P_{n}$ at this endpoint. As a consequence, we obtain sharp error bounds for the recurrence.

Key words. Legendre polynomials, three-term recurrence, floating-point arithmetic

AMS subject classifications. 65D20, 65Q30, 33F05

\section{Introduction.}

1.1. Main results. A new method of analysis of three-term recurrences in floating-point arithmetic was introduced in [4]. That paper is devoted to the numerical evaluation of the Chebyshev polynomials $T_{n}$ by a three-term recurrence. Approximate values of Chebyshev polynomials $T_{n}$ computed in floating-point arithmetic are denoted by $\widehat{T}_{n}$. It is shown in [4, Theorem 1] that in a neighborhood of an endpoint of the interval $[-1,1], \widehat{T}_{n}$ agrees with the line tangent to $T_{n}$ at that endpoint. New error estimates are derived from this observation.

Our objective in this paper is to extend this approach to the case of Legendre polynomials. For $z \in \mathbb{R}$, the Legendre polynomials $P_{n}$ are defined by the three-term recurrence

$$
P_{n}(z)=\frac{2 n-1}{n} z P_{n-1}(z)-\frac{n-1}{n} P_{n-2}(z), \quad n=2,3, \ldots,
$$

with $P_{0}(z)=1$ and $P_{1}(z)=z$. Approximate values of the Legendre polynomials computed by the recurrence in floating-point arithmetic are denoted by $\widehat{P}_{n}$; see Section 2 for a definition. Throughout this paper, we use a floating-point numbers system $\mathbb{F}$ with the unit roundoff $u$.

Our main tool is described in Theorem 3.1. We show there that if $x \in \mathbb{F}$ and

$$
|x-1|<\frac{\sqrt{u}}{\sqrt{n(n+1)}},
$$

then

$$
\widehat{P}_{n}(x)=P_{n}(1)+P_{n}^{\prime}(1)(x-1)=1+\frac{1}{2} n(n+1)(x-1) .
$$

Equivalently, the floating-point approximation $\widehat{P}_{n}(x)$ coincides with the line tangent to $P_{n}$ at $x_{0}=1$. As a consequence, we derive new upper and lower error bounds for $\widehat{P}_{n}$ valid in a neighborhood of the endpoints. Specifically, we prove in Theorem 5.1 that if $n \geqslant 1$, $n(n+1)<\frac{1}{u}$, and

$$
1-\frac{\sqrt{u}}{\sqrt{n(n+1)}}<x \leqslant 1 \text {, }
$$

*Received May 5, 2017. Accepted March 18, 2021. Published online on April 20, 2021. Recommended by Bernd Beckermann.

$\dagger$ Acoustics Research Institute, Austrian Academy of Sciences, Wohllebengasse 12-14, 1040 Vienna, Austria (tomasz.hrycak@oeaw.ac.at, sschmutzhard@kfs.oeaw.ac.at). 
then

$$
\frac{17}{36} P_{n}^{\prime \prime}(1) \cdot(1-x)^{2} \leqslant P_{n}(x)-\widehat{P}_{n}(x) \leqslant \frac{1}{2} P_{n}^{\prime \prime}(1) \cdot(1-x)^{2} .
$$

As a consequence, we show in Theorem 5.2 that if $n \geqslant 2$ and $n(n+1)<\frac{1}{u}$, then there exists $x \in \mathbb{F}$ such that

$$
P_{n}(x)-\widehat{P}_{n}(x) \geqslant \frac{u n^{2}}{68} .
$$

This result is optimal up to a constant factor since a corresponding upper bound of the form $\mathcal{O}\left(u n^{2}\right)$ is given in [6, Theorem 4.1].

Combining the lower bound from Theorem 5.2 and an upper bound from [6, Theorem 4.1], we infer that the absolute error $\left|\widehat{P}_{n}-P_{n}\right|$ is maximized close to the endpoints of the interval $[-1,1]$. Specifically, the maximum absolute error is located within a distance of $\mathcal{O}\left(\frac{1}{n^{2}}\right)$ from the endpoints; see Theorem 5.3.

1.2. Previous work. We outline recent advances in the numerical evaluation of Legendre polynomials on the interval $[-1,1]$. The three-term recurrence is the method of choice when all Legendre polynomials up to a certain degree are needed. On the other hand, the authors of [1] consider the evaluation of a single Legendre polynomial. Such an approach is used, e.g., for constructing Gauss-Legendre quadratures or for the summation of Legendre series in parallel. The authors describe an evaluation algorithm that combines asymptotic formulas with approximations using tabulated zeros of Legendre polynomials and which has time complexity of $\mathcal{O}(1)$. A similar method is developed in [2] with the focus on the construction of Gauss-Jacobi quadratures.

In [7], the authors propose an algorithm for the evaluation of Legendre polynomials in arbitrary precision using midpoint-radius interval arithmetic. Subsequently, they construct Gauss-Legendre quadratures by means of the interval Newton method, which provides rigorous enclosures of the quadrature nodes and weights. The algorithm is designed for precisions of hundreds to thousands of bits. It combines approximations by hypergeometric series, asymptotic expansions, and the three-term recurrence (1.1).

An upper error bound for the three-term recurrence (2.3)-(2.4) on the interval $[-1,1]$ is derived in [6]. This upper bound lies within a constant from the lower bound given in (1.3).

The inequalities (1.2), (1.3) and Theorem 5.3 have their counterparts in the case of Chebyshev polynomials [4].

2. Floating-point arithmetic. A straightforward way to implement (1.1) in floatingpoint arithmetic is given by

$$
\begin{aligned}
\widetilde{P}_{n}(x)= & ((2 \otimes n \ominus 1) \oslash n) \otimes\left(x \otimes \widetilde{P}_{n-1}(x)\right) \ominus \\
& ((n \ominus 1) \oslash n) \otimes \widetilde{P}_{n-2}(x), \quad n=2,3, \ldots,
\end{aligned}
$$

with $\widetilde{P}_{0}(x)=1$ and $\widetilde{P}_{1}(x)=x, x \in \mathbb{F}$. Circles indicate rounded-to-nearest arithmetic operations with gradual underflow. In the absence of overflow, the results of circled operations are in $\mathbb{F}$.

Unfortunately, the so defined $\widetilde{P}_{n}$ is irregular and difficult to analyze. For example, in IEEE single precision, we have $\widetilde{P}_{0}(1)=\widetilde{P}_{1}(1)=\widetilde{P}_{2}(1)=1$. However,

$$
\widetilde{P}_{3}(1)=(5 \oslash 3) \ominus(2 \oslash 3)=\left(\frac{5}{3}-\frac{2}{3} u_{s}\right) \ominus\left(\frac{2}{3}+\frac{1}{3} u_{s}\right)=1-u_{s},
$$


where $u_{s}=2^{-24}$ is the unit roundoff in single precision.

Instead of $\widetilde{P}_{n}$, we study the following approximation of $P_{n}$ :

$$
\begin{aligned}
\widehat{P}_{n}(x)= & \left(2 \otimes\left(x \otimes \widehat{P}_{n-1}(x)\right) \ominus \widehat{P}_{n-2}(x)\right) \ominus \\
& \left(x \otimes \widehat{P}_{n-1}(x) \ominus \widehat{P}_{n-2}(x)\right) \oslash n, \quad n=2,3, \ldots
\end{aligned}
$$

with $\widehat{P}_{0}(x)=1$ and $\widehat{P}_{1}(x)=x, x \in \mathbb{F}$. Clearly, $\widehat{P}_{n}(1)=1$ as long as $\widehat{P}_{n}(1)$ is defined. One can consider this approach a perturbation of a three-term recurrence satisfied by Chebyshev polynomials. It can be shown [4, Lemma 5] that

$$
2 \otimes\left(x \otimes \widehat{P}_{n-1}(x)\right)=(2 \otimes x) \otimes \widehat{P}_{n-1}(x)
$$

if no floating-point exception occurs. Since we must compute $x \otimes \widehat{P}_{n-1}(x)$ in (2.4) anyway, we reuse this quantity in (2.3) in order to reduce the operation count. Throughout this paper, we evaluate $\widehat{P}_{n}$ and $\widetilde{P}_{n}$ only at numbers $x \in \mathbb{F}$. Experiments presented in Section 6 indicate that $\widetilde{P}_{n}$ and $\widehat{P}_{n}$ approximate $P_{n}$ with comparable accuracy.

We assume that floating-point numbers in $\mathbb{F}$ have base 2 and $t$-digit significands [3, Equation (2.1)]. We refer to the elements of $\mathbb{F}$ as representable numbers. The unit roundoff is given by $u=2^{-t}\left[3\right.$, p. 42]. We assume that $\frac{1}{2}, 1,2,4 \in \mathbb{F}$. Consequently, $u \leqslant \frac{1}{2}$. Additionally, we assume that $u \in \mathbb{F}$. Our assumptions on $\mathbb{F}$ are satisfied for IEEE single and double precision arithmetic.

Finally, we assume that the degrees $n$ of the Legendre polynomials appearing in (2.4) are representable. In the remainder of this section, we present results that are used in Section 3.

LEMMA 2.1. If $x \in \mathbb{F}$ and $\frac{1}{2} \leqslant x \leqslant 2$, then $x-1 \in \mathbb{F}$.

Proof. Since $u \in \mathbb{F}$, all integer multiples of $u$ in the interval $[-1,1]$ are representable. If $\frac{1}{2} \leqslant x \leqslant 1$, then $x$ is an integer multiple of $u$. If $1 \leqslant x \leqslant 2$, then $x=1+2 u k$ for some integer $k$, and again $x$ is an integer multiple of $u$. Consequently, $x-1$ is an integer multiple of $u$ lying in the interval $[-1,1]$, and thus $x-1 \in \mathbb{F}$.

Lemma 2.2. Let $r \in \mathbb{Z}, 2^{r} u, 2^{r}, x \in \mathbb{F}$, and $2^{r-1} \leqslant x \leqslant 2^{r}$. If $a, b \in \mathbb{Z}$ and $\left|a x+2^{r} u b\right| \leqslant 2^{r}$, then $a x+2^{r} u b \in \mathbb{F}$.

Proof. Since $2^{r} u$ and $2^{r} \in \mathbb{F}$, all integer multiples of $2^{r} u$ with modulus not exceeding $2^{r}$ are representable. Since $x \in \mathbb{F}$ and $2^{r-1} \leqslant x \leqslant 2^{r}$, we have $x=2^{r} u k$ for some $k \in \mathbb{Z}$. Thus, $a x+2^{r} u b=(a k+b) 2^{r} u$ is an integer multiple of $2^{r} u$. The claim follows from the assumption that $\left|a x+2^{r} u b\right| \leqslant 2^{r} \in \mathbb{F}$.

LEMMA 2.3. If $n \geqslant 2$ is an integer, $x \in \mathbb{F}$, and $1-\frac{1}{n} \leqslant x \leqslant 1+\frac{2}{n}$, then $n(x-1) \in \mathbb{F}$.

Proof. If $1-\frac{1}{n} \leqslant x \leqslant 1$, then $\frac{1}{2} \leqslant x \leqslant 1$ and $|n(x-1)| \leqslant 1$. The claim follows from Lemma 2.2 applied with $r=0, a=n$, and $b=-n / u$. If $1 \leqslant x \leqslant 1+\frac{2}{n}$, then $1 \leqslant x \leqslant 2$ and $|n(x-1)| \leqslant 2$. The claim follows from Lemma 2.2 applied with $r=1, a=n$, and $b=-n /(2 u)$.

The following two lemmas are proved in [4, Lemma 3 and Lemma 4].

LEMMA 2.4. If $N \geqslant 1$ is an integer, $u$ is the unit roundoff, $x \in \mathbb{F}$, and

$$
|x-1|<\frac{\sqrt{u}}{\sqrt{2 N}}
$$

then

$$
|x-1| \leqslant \frac{1}{2 N+2}
$$


LEMMA 2.5. If $N \geqslant 1$ is an integer, $u$ is the unit roundoff, $x \in \mathbb{F}$, and

$$
|x-1|<\frac{\sqrt{u}}{\sqrt{2 N}}
$$

then $1+N(x-1) \in \mathbb{F}, 1+(N+1)(x-1) \in \mathbb{F}$ and

$$
x \otimes(1+N(x-1))=1+(N+1)(x-1) .
$$

No floating-point exception occurs in (2.5).

3. Representation of $\widehat{\boldsymbol{P}}_{\boldsymbol{n}}$ as a tangent line. In this section, we derive representations of $\widehat{P}_{n}(x)$ as tangent lines to the Legendre polynomial $P_{n}$ at the endpoints of the interval $[-1,1]$.

THEOREM 3.1. If $n \geqslant 2$ is an integer, $u$ is the unit roundoff, $x \in \mathbb{F}$, and

$$
|x-1|<\frac{\sqrt{u}}{\sqrt{n(n+1)}}
$$

then

$$
\widehat{P}_{n}(x)=P_{n}(1)+P_{n}^{\prime}(1)(x-1)=1+\frac{1}{2} n(n+1)(x-1) .
$$

We note that the relations $P_{n}(1)=1$ and $P_{n}^{\prime}(1)=\frac{1}{2} n(n+1)$ follow from (4.3) and (4.4), respectively. Since $\widehat{P}_{0}(x)=1$ and $\widehat{P}_{1}(x)=x$ by definition, if $n=0$ or $n=1$, then (3.2) holds for every $x \in \mathbb{F}$.

Proof. We proceed by induction with respect to $n$. The claim (3.2) is true for $n=0$ and $n=1$. Let us assume that (3.2) holds for $n-2$ and $n-1$. Our approach is to explicitly compute the terms appearing in (2.3) and (2.4) and then to verify (3.2). The assumption (3.1) justifies our use of Lemmas 2.4 and 2.5 whenever $1 \leqslant N \leqslant \frac{1}{2} n(n+1)$.

From Lemma 2.4 applied with $N=\frac{1}{2} n(n+1)$, we deduce that

$$
|x-1| \leqslant \frac{1}{n^{2}+n+2} .
$$

Using the induction hypothesis for $\widehat{P}_{n-1}(x)$ and Lemma 2.5 with $N=\frac{1}{2}(n-1) n$, we obtain

(3.4) $x \otimes \widehat{P}_{n-1}(x)=x \otimes\left(1+\frac{1}{2}(n-1) n \cdot(x-1)\right)=1+\left[\frac{1}{2}(n-1) n+1\right](x-1)$.

Combining this with (3.3), we see that

$$
\left|x \otimes \widehat{P}_{n-1}(x)\right| \leqslant 1+\left[\frac{1}{2}(n-1) n+1\right] \frac{1}{n^{2}+n+2}<\frac{3}{2} .
$$

Consequently, no overflow occurs when computing $2 \otimes\left(x \otimes \widehat{P}_{n-1}(x)\right)$, and thus

$$
2 \otimes\left(x \otimes \widehat{P}_{n-1}(x)\right)=2+\left(n^{2}-n+2\right)(x-1) .
$$

From the induction hypothesis for $\widehat{P}_{n-2}(x)$, we obtain

$$
\begin{aligned}
& 2 \otimes\left(x \otimes \widehat{P}_{n-1}(x)\right)-\widehat{P}_{n-2}(x)= \\
& =2+\left(n^{2}-n+2\right)(x-1)-\left[1+\frac{1}{2}(n-2)(n-1)(x-1)\right] \\
& =1+\left[\frac{1}{2} n(n+1)+1\right](x-1) .
\end{aligned}
$$


From Lemma 2.5 used with $N=\frac{1}{2} n(n+1)$, we deduce that the number appearing in (3.5) is representable. Consequently,

$$
2 \otimes\left(x \otimes \widehat{P}_{n-1}(x)\right) \ominus \widehat{P}_{n-2}(x)=1+\left[\frac{1}{2} n(n+1)+1\right](x-1) .
$$

If follows from (3.4) and the induction hypothesis for $\widehat{P}_{n-2}(x)$ that

$$
\begin{aligned}
& x \otimes \widehat{P}_{n-1}(x)-\widehat{P}_{n-2}(x)= \\
& =1+\left[\frac{1}{2}(n-1) n+1\right](x-1)-\left[1+\frac{1}{2}(n-2)(n-1)(x-1)\right] \\
& =n(x-1) .
\end{aligned}
$$

In view of (3.3), from Lemma 2.1 and Lemma 2.3, respectively, we conclude that the numbers $x-1$ and $n(x-1)$ are representable. Therefore, (3.7)-(3.9) imply that

$$
\left(x \otimes \widehat{P}_{n-1}(x) \ominus \widehat{P}_{n-2}(x)\right) \oslash n=n(x-1) \oslash n=x-1 .
$$

Substituting (3.6) and (3.10) into (2.3)-(2.4), we obtain

$$
\widehat{P}_{n}(x)=\left(1+\left[\frac{1}{2} n(n+1)+1\right](x-1)\right) \ominus(x-1) .
$$

Applying Lemma 2.5 with $N=\frac{1}{2} n(n+1)-1$, we find that the number $1+\frac{1}{2} n(n+1)(x-1)$ is representable. Thus, the right-hand side of (3.11) evaluates to $1+\frac{1}{2} n(n+1)(x-1)$, and the induction proof of (3.2) is complete.

In view of symmetry, an analogous result is valid in the neighborhood of $x=-1$.

THEOREM 3.2. If $n \geqslant 1$ is an integer, $u$ is the unit roundoff, $x \in \mathbb{F}$, and

$$
|x+1|<\frac{\sqrt{u}}{\sqrt{n(n+1)}},
$$

then

$$
\widehat{P}_{n}(x)=(-1)^{n}\left(1-\frac{1}{2} n(n+1)(x+1)\right) .
$$

4. Properties of Legendre polynomials. We present some properties of Legendre polynomials that are used in Section 5. We denote the $m$ th derivative of the Legendre polynomial $P_{n}$ by $P_{n}^{(m)}, m, n=0,1, \ldots$ For $\alpha, \beta>-1$, we denote the Jacobi polynomial of degree $n$ by $P_{n}^{(\alpha, \beta)}[8,18.5 .8]$. For $z \in \mathbb{C}$, Pochhammer's symbol is defined as follows $[8,5.2 .4]$ :

$$
(z)_{n}=z(z+1)(z+2) \cdots(z+n-1), \quad(z)_{0}=1 .
$$

In particular, if $p$ and $q$ are non-negative integers, then

$$
(p+1)_{q}=\frac{(p+q) !}{p !}
$$

LEMMA 4.1. If $n$ and $m$ are integers with $0 \leqslant m \leqslant n$, then

$$
\begin{aligned}
P_{n}^{(m)} & =2^{-m} \frac{(n+m) !}{n !} P_{n-m}^{(m, m)}, \\
\left|P_{n}^{(m)}(z)\right| & \leqslant P_{n}^{(m)}(1)=2^{-m} \frac{(n+m) !}{m !(n-m) !}, \quad-1 \leqslant z \leqslant 1 .
\end{aligned}
$$


Proof. Since $P_{n}=P_{n}^{(0,0)}$, cf. [8, 18.7.9], (4.1) follows by induction from a differentiation formula for Jacobi polynomials $[8,18.9 .15]$

$$
\left(P_{n}^{(\alpha, \alpha)}\right)^{\prime}=\frac{1}{2}(n+2 \alpha+1) P_{n-1}^{(\alpha+1, \alpha+1)} .
$$

Specifically,

$$
\left(P_{n}^{(0,0)}\right)^{(m)}=\frac{1}{2}(n+1)\left(P_{n-1}^{(1,1)}\right)^{(m-1)}=\ldots=\frac{1}{2^{m}}(n+1)(n+2) \ldots(n+m) P_{n-m}^{(m, m)} .
$$

The following inequality, which holds for $-1 \leqslant z \leqslant 1$, is a special case of $[8,18.14 .1]$ :

$$
\left|P_{n-m}^{(m, m)}(z)\right| \leqslant P_{n-m}^{(m, m)}(1)=\frac{(m+1)_{n-m}}{(n-m) !}=\frac{n !}{m !(n-m) !} .
$$

Combining this with (4.1), we obtain (4.2).

For the particular values $m=0,1,2,3$, the lemma implies that the following inequalities are valid for $-1 \leqslant z \leqslant 1$ and $n=0,1, \ldots$

$$
\begin{aligned}
\left|P_{n}(z)\right| \leqslant P_{n}(1) & =1, \\
\left|P_{n}^{\prime}(z)\right| \leqslant P_{n}^{\prime}(1) & =\frac{1}{2} n(n+1), \\
\left|P_{n}^{\prime \prime}(z)\right| \leqslant P_{n}^{\prime \prime}(1) & =\frac{1}{8}(n-1) n(n+1)(n+2), \\
\left|P_{n}^{\prime \prime \prime}(z)\right| \leqslant P_{n}^{\prime \prime \prime}(1) & =\frac{1}{48}(n-2)(n-1) n(n+1)(n+2)(n+3) .
\end{aligned}
$$

5. Error bounds for the three-term recurrence. In this section, we derive upper and lower error bounds for the absolute error of $\widehat{P}_{n}$. These estimates are valid in a neighborhood of the endpoints and rely on Theorem 3.1.

THEOREM 5.1. If $n \geqslant 1$, $u$ is the unit roundoff, $n(n+1)<\frac{1}{u}, x \in \mathbb{F}$, and

$$
1-\frac{\sqrt{u}}{\sqrt{n(n+1)}}<x \leqslant 1
$$

then

$$
\frac{17}{36} P_{n}^{\prime \prime}(1) \cdot(1-x)^{2} \leqslant P_{n}(x)-\widehat{P}_{n}(x) \leqslant \frac{1}{2} P_{n}^{\prime \prime}(1) \cdot(1-x)^{2} .
$$

The first inequality in (5.2) and its proof have been proposed by the reviewer. A similar estimate can be deduced from [5, Equation (3)].

Proof. Using (3.2) and the Taylor series of $P_{n}$ with two terms, we obtain

$$
\begin{aligned}
P_{n}(x)-\widehat{P}_{n}(x) & =P_{n}(x)-\left[P_{n}(1)+P_{n}^{\prime}(1)(x-1)\right] \\
& =\frac{1}{2} P_{n}^{\prime \prime}(\xi)(x-1)^{2} \leqslant \frac{1}{2} P_{n}^{\prime \prime}(1)(1-x)^{2},
\end{aligned}
$$

where $x \leqslant \xi \leqslant 1$, and the last inequality follows from (4.5). Inequality (5.1) yields that

$$
0 \leqslant 1-x<\frac{\sqrt{u}}{\sqrt{n(n+1)}}<\frac{1}{n(n+1)} .
$$


Combining (3.2) and the Taylor series of $P_{n}$ with three terms, we infer that

$$
\begin{aligned}
P_{n}(x)-\widehat{P}_{n}(x) & =\frac{1}{2} P_{n}^{\prime \prime}(1)(x-1)^{2}+\frac{1}{6} P_{n}^{\prime \prime \prime}(\zeta)(x-1)^{3} \\
& \geqslant \frac{1}{2} P_{n}^{\prime \prime}(1)(1-x)^{2}-\frac{1}{6} P_{n}^{\prime \prime \prime}(1)(1-x)^{3},
\end{aligned}
$$

where $x \leqslant \zeta \leqslant 1$ and the last inequality follows from (4.6). Substituting (4.5), (4.6), and (5.3) into (5.4), we obtain

$$
P_{n}(x)-\widehat{P}_{n}(x) \geqslant \frac{1}{2} P_{n}^{\prime \prime}(1)(1-x)^{2}\left(1-\frac{(n-2)(n+3)}{18 n(n+1)}\right) \geqslant \frac{17}{36} P_{n}^{\prime \prime}(1)(1-x)^{2} .
$$

The following theorem studies an extremal case of the estimate (5.2).

THEOREM 5.2. Let $n \geqslant 2$ be an integer, $u$ be the unit roundoff, and $x$ be the smallest representable number such that

$$
x>1-\frac{\sqrt{u}}{\sqrt{n(n+1)}} .
$$

If $n(n+1)<\frac{1}{u}$, then

$$
P_{n}(x)-\widehat{P}_{n}(x) \geqslant \frac{u n^{2}}{68}
$$

Proof. The assumption $n(n+1)<\frac{1}{u}$ implies that

$$
1-\frac{\sqrt{u}}{\sqrt{n(n+1)}}<1-u \text {. }
$$

Since $1-u$ is representable, (5.7) implies that $x \leqslant 1-u$. From (5.5) and the assumptions $u \leqslant \frac{1}{2}$ and $n \geqslant 2$, we deduce that $x>1-\frac{1}{\sqrt{12}}>\frac{1}{2}$. All representable numbers on the interval $\left[\frac{1}{2}, 1\right]$ are integer multiples of $u$. Thus, $x=1-k u$ for some integer $k \geqslant 1$. From the definition of $x$, it follows that

$$
1-\frac{\sqrt{u}}{\sqrt{n(n+1)}} \geqslant x-u=1-(k+1) u .
$$

Consequently,

$$
(k+1) u \geqslant \frac{\sqrt{u}}{\sqrt{n(n+1)}}
$$

and

$$
1-x=\frac{k}{k+1} \cdot(k+1) u \geqslant \frac{1}{2} \cdot \frac{\sqrt{u}}{\sqrt{n(n+1)}} .
$$

Substituting this into (5.2) and using (4.5), we obtain

$$
P_{n}(x)-\widehat{P}_{n}(x) \geqslant \frac{17}{36 \cdot 32}(n-1)(n+2) u \geqslant \frac{u n^{2}}{68} .
$$


Combining Theorem 5.2 with an upper error bound shown in [6, Theorem 4.1], we deduce that the absolute error $\left|\widehat{P}_{n}-P_{n}\right|$ is maximized within a distance of $\mathcal{O}\left(\frac{1}{n^{2}}\right)$ from the endpoints of the interval $[-1,1]$.

THEOREM 5.3. Let $x \in \mathbb{F}$ be a point where the maximum of the error $\left|\widehat{P}_{n}(x)-P_{n}(x)\right|$ on the interval $[-1,1]$ occurs. If $2 \leqslant n \leqslant \frac{1}{5 \sqrt{u}}$, then

$$
1-|x| \leqslant \frac{7.7 \cdot 10^{7}}{n^{2}} \text {. }
$$

Proof. First, since $\widehat{P}_{n}(1)=P_{n}(1)=1$ and $\widehat{P}_{n}(-1)=P_{n}(-1)=(-1)^{n}$, we obtain that $|x|<1$. From Theorem 5.2 and [6, Equation (4.2)], we infer that

$$
\frac{u n^{2}}{68} \leqslant \max _{y \in \mathbb{F} \cap[-1,1]}\left|\widehat{P}_{n}(y)-P_{n}(y)\right|=\left|\widehat{P}_{n}(x)-P_{n}(x)\right| \leqslant \frac{129 u n}{\sqrt{1-x^{2}}} .
$$

Consequently,

$$
1-|x| \leqslant 1-x^{2} \leqslant \frac{68^{2} \cdot 129^{2}}{n^{2}} \leqslant \frac{7.7 \cdot 10^{7}}{n^{2}}
$$

We note that $\left[6\right.$, Theorem 4.1] requires the restriction $n \leqslant \frac{1}{5 \sqrt{u}}$, while Theorem 5.2 has the weaker assumption of $n(n+1)<\frac{1}{u}$.

Our numerical simulations indicate that the constant in (5.8) is overly pessimistic; see Section 6.

6. Numerical experiments. We have conducted numerical experiments in order to validate Theorem 5.1 and to compare the accuracy of the recurrences (2.1)-(2.2) and (2.3)(2.4). Our experiments have been performed in GNU Octave, version 4.0.1.

We denote by $\widetilde{P}_{n}^{s}$ and $\widehat{P}_{n}^{s}$ the approximate values of the Legendre polynomial $P_{n}$ computed in single precision by the recurrences (2.1)-(2.2) and (2.3)-(2.4), respectively. Similarly, $\widetilde{P}_{n}^{d}$ denotes the approximation obtained in double precision using (2.1)-(2.2). For a fixed degree $n \geqslant 2$, the Legendre polynomial $P_{n}$ is evaluated at all representable points in the interval $\left(1-\frac{\sqrt{u}}{\sqrt{n(n+1)}}, 1\right]$, where $u=2^{-24}$ is the unit roundoff in single precision. All representable points in this interval are integer multiples of $u$. The errors of the evaluation in single precision are found by comparing $\widehat{P}_{n}^{s}$ and $\widehat{P}_{n}^{s}$ with $\widetilde{P}_{n}^{d}$. Specifically, the maximum absolute errors are defined as

$$
\begin{aligned}
& \widetilde{e}_{n}=\max _{k}\left|\widetilde{P}_{n}^{s}(k u)-\widetilde{P}_{n}^{d}(k u)\right|, \\
& \widehat{e}_{n}=\max _{k}\left|\widehat{P}_{n}^{s}(k u)-\widetilde{P}_{n}^{d}(k u)\right|,
\end{aligned}
$$

where the maximum is taken over all integers $k$ such that $1-\frac{\sqrt{u}}{\sqrt{n(n+1)}}<k u \leqslant 1$. Because of symmetry, one does not need to compute errors at negative arguments.

Figure 6.1 displays the maximum absolute errors of both methods applied to the Legendre polynomials of degrees $n=2,4,8, \ldots, 1024$. The lower bound $\frac{u n^{2}}{68}$ appearing in (5.6) is represented by white circles. We observe that both methods have comparable accuracy.

Additionally, we evaluate the Legendre polynomials at all integer multiples of $u$ in the interval $[0,1]$. We observe that the maximum absolute error of $\widetilde{P}_{n}$ is attained within $\frac{0.73}{n^{2}}$ from the endpoint $x=1$, while the maximum absolute error of $\widehat{P}_{n}$ is attained within $\frac{0.5}{n^{2}}$ from this endpoint. While not all representable points from the interval $\left[0, \frac{1}{2}\right]$ are included, this experiment indicates that (5.8) holds with a much smaller constant. 


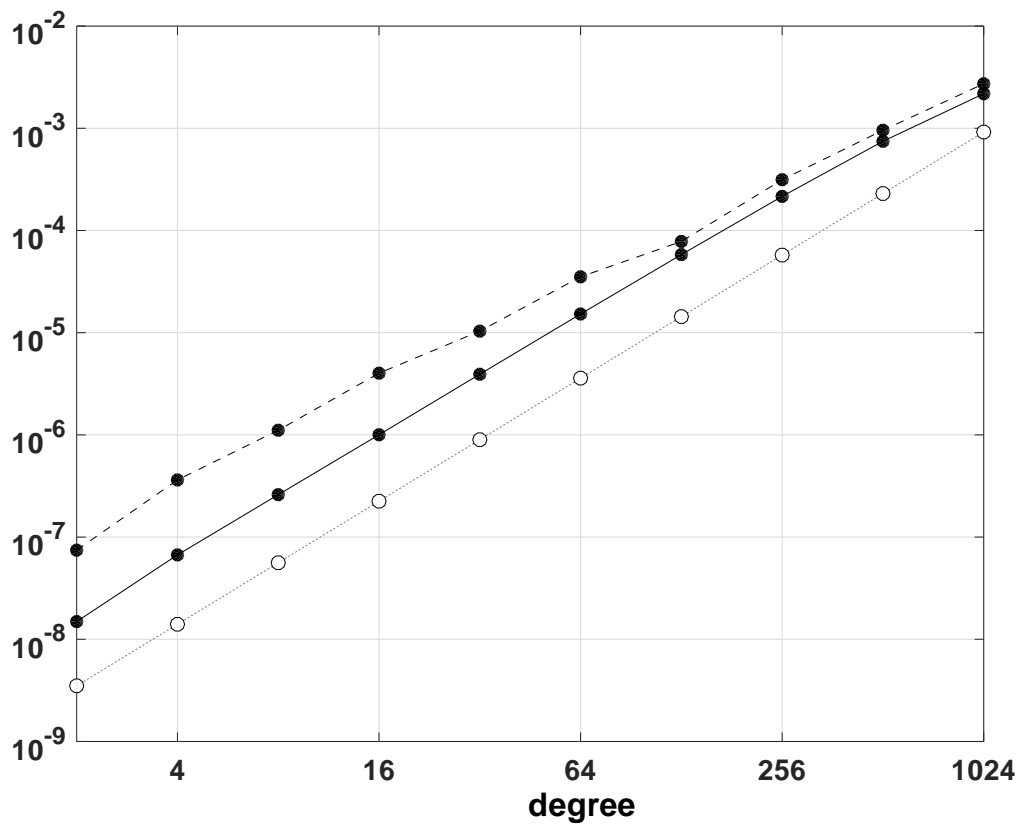

FIG. 6.1. The maximum absolute errors of $\widetilde{P}_{n}$ (dashed line) and $\widehat{P}_{n}$ (solid line) for $n=2,4,8, \ldots, 1024$. The lower bound $\frac{u n^{2}}{68}$ appearing in (5.6) is represented by white circles.

7. Conclusions. For a fixed integer $n \geqslant 0$, let $L_{n}(x):=1+\frac{1}{2} n(n+1)(x-1)$ be the tangent line to the Legendre polynomial $P_{n}$ at 1 . Since $L_{n}(1)=1$ and $L_{n}^{\prime}(1)=\frac{1}{2} n(n+1)$ is a non-negative integer, it holds that in a sufficiently small neighborhood of 1 , the mapping $x \mapsto L_{n}(x)$ takes $\mathbb{F}$ into $\mathbb{F}$. Consequently, $L_{n}(x)$ is the closest representable number to $P_{n}(x)$ if $x$ is sufficiently close to 1 . In view of Theorem 3.1 , we have $\widehat{P}_{n}(x)=L_{n}(x)$ in a possibly smaller neighborhood. However, by methods similar to those used in the proof of Theorem 5.1, one can show that both in single and in double precision, there exists $x \in \mathbb{F}$ such that $\widehat{P}_{n}(x)=L_{n}(x)$, while $\widehat{P}_{n}(x)$ and $P_{n}(x)$ are separated by several representable numbers.

Acknowledgments. We are grateful for comments made by the editor and the reviewer, and their many helpful suggestions. The authors are supported by the Innovationsfonds "Forschung, Wissenschaft und Gesellschaft" of the Austrian Academy of Sciences on the project "Railway vibrations from tunnels".

\section{REFERENCES}

[1] I. Bogaert, B. Michiels, AND J. Fostier, O(1) computation of Legendre polynomials and Gauss-Legendre nodes and weights for parallel computing, SIAM J. Sci. Comput., 34 (2012), pp. C83-C101.

[2] N. HALE AND A. TOWNSEND, Fast and accurate computation of Gauss-Legendre and Gauss-Jacobi quadrature nodes and weights, SIAM J. Sci. Comput., 35 (2013), pp. A652-A674.

[3] N. J. Higham, Accuracy and Stability of Numerical Algorithms, 2nd ed., SIAM, Philadelphia, 2002.

[4] T. HRYCAK AND S. SCHMUTZHARD, Evaluation of Chebyshev polynomials by a three-term recurrence in floating-point arithmetic, BIT, 58 (2018), pp. 317-330.

[5] - Inequalities involving Gegenbauer polynomials and their tangent lines, Math. Inequal. Appl., 22 (2019), pp. 353-360. 
ETNA

Kent State University and

Johann Radon Institute (RICAM)

[6] - Evaluation of Legendre polynomials by a three-term recurrence in floating-point arithmetic, IMA J. Numer. Anal., 40 (2020), pp. 587-605.

[7] F. JohansSOn AND M. MeZZARobBa, Fast and rigorous arbitrary-precision computation of Gauss-Legendre quadrature nodes and weights, SIAM J. Sci. Comput., 40 (2018), pp. C726-C747.

[8] F. W. Olver, D. W. Lozier, R. F. Boisvert, AND C. W. Clark, The NIST Handbook of Mathematical Functions, Cambridge University Press, New York, 2010. 